\title{
Correction to: Selective Immunomodulatory and Neuroprotective Effects of a NOD2 Receptor Agonist on Mouse Models of Multiple Sclerosis
}

\author{
Adham Fani Maleki ${ }^{1}$. Giulia Cisbani ${ }^{1} \cdot$ Nataly Laflamme $^{1} \cdot$ Paul Prefontaine $^{1} \cdot$ Marie-Michele Plante $^{1}$. \\ Joanie Baillargeon ${ }^{1} \cdot$ Manu Rangachari $^{1} \cdot$ Jean Gosselin ${ }^{2} \cdot$ Serge Rivest $^{1}$
}

Published online: 27 April 2021

(c) The American Society for Experimental NeuroTherapeutics, Inc. 2021

https://doi.org/10.1007/s13311-020-00998-0

This article was corrected to add the captions to the supplementary figures.

Publisher's Note Springer Nature remains neutral with regard to jurisdictional claims in published maps and institutional affiliations.

The original article can be found online at https://doi.org/10.1007/ s13311-020-00998-0

Serge Rivest

serge.rivest@crchudequebec.ulaval.ca

1 Neuroscience Laboratory, CHU of Quebec Research Center and Department of Molecular Medicine, Faculty of Medicine, Laval University, 2705 Laurier Boul., Quebec City, QC G1V 4G2, Canada

2 Laboratory of Innate Immunity, CHU of Quebec Research Center and Department of Molecular Medicine, Faculty of Medicine, Laval University, Laval University, 2705 Laurier Boul., Quebec City, QC G1V 4G2, Canada 